\title{
Cryptococcus as a cause of proliferative glomerulonephritis in an immunodeficient HIV+ patient
}

\author{
Betul Celik, Tangul Bulut, Arzu D. Yalcin \\ SB University Antalya Hospital, Antalya, Turkey
}

\begin{abstract}
This report presents the first proliferative glomerulonephritis (GN) induced by Cryptococcus neoformans in a human immunodeficiency virus (HIV)+ patient. While the patient was on medication against HBV, HIV RNA was found $4.25 \times 10^{5}$ in his blood. Six months later, he experienced headache, visual blurring, nausea, and vomiting and was hospitalised due to possible CMV, TBC, or HIV-related retinopathy and raised creatinine level. Cryptococcus neoformans grew in his blood culture. Albumin was 2.2 at that time. Because of the persistent proteinuria, kidney biopsy was applied. On histological examination, some glomeruli appeared normal, while endocapillary proliferative glomerulonephritis was seen in other glomeruli. Tubules were enlarged with many round yeasts. Mucicarmine stain confirmed peripheral capsule. Immunofluorescence staining with IgG, IgA, IgM, C3, C1q, fibrinogen, kappa, and lambda light chains revealed no deposition. Electron microscopy was not applied. The patient was well with $26 \mathrm{BUN}$ (range $8-20 \mathrm{mg} / \mathrm{dl}$ ) and 1.70 creatinin (range $0.84-1.25 \mathrm{mg} / \mathrm{dl}$ ) five months after the biopsy date.

The most common secondary immunodeficiency is caused by HIV, and although different forms of renal disease were noted in this population, HIV-associated nephropathy (HIVAN) is the most common cause of renal failure in HIV1+ patients. The term HIVAN is reserved for the typical histopathological form of focal and segmental glomerulosclerosis. Cryptococcal GN was not reported in the glomeruli previously.
\end{abstract}

HIV AIDS Rev 2017; 16: 61-63 DOI: https://doi.org/10.5114/hivar.2017.65222

Key words: HIV, Cryptococcus, glomerulonephritis.

\section{Introduction}

Human immunodeficiency virus (HIV) causes glomerulonephritis, which is called HIV-associated nephropathy (HIVAN), in which the proliferative feature is exclusive for parietal epithelial cells. Proliferative glomerulonephritis caused by Cryptococcus has never been reported in the literature.

\section{Case description}

A 43-year-old male patient complained of weakness, fatigue, and weight loss (20 kg in the preceding five months) in May 2015 and was found to be hepatitis B positive. Subsequent liver biopsy revealed 3/6 fibrosis and 8/18 HAI score. While the patient was on medication against HBV, HIV RNA was found $4.25 \times 10^{5}$ in July. In December 2015 he experi-



Article history:

Received: 24.10 .2016

Received in revised form: 15.11.2016

Accepted: 17.11.2016

Available online: 19.01.2017
International Journal of HIV-Related Problems

HIV \& AIDS

R e v i e w 


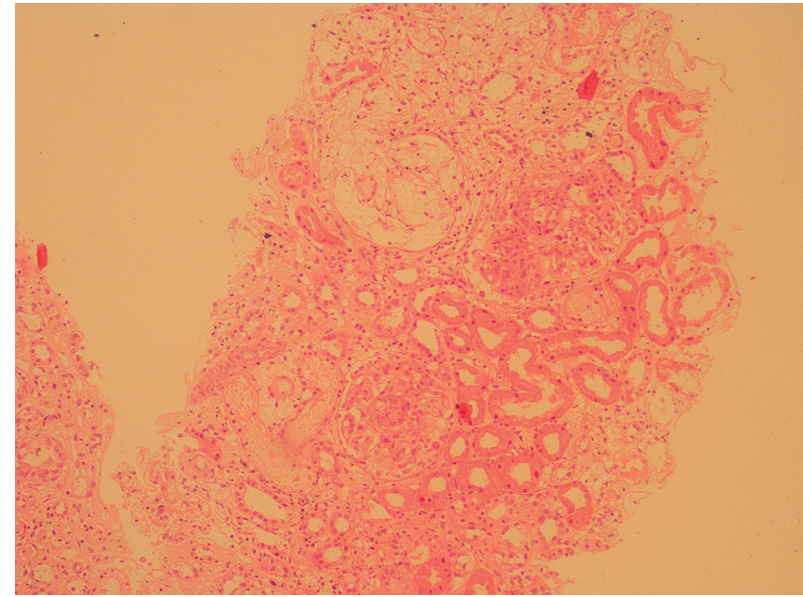

Figure 1. Three glomeruli in which two of them have endocapillary proliferation and tubular focus $(\mathrm{H} / \mathrm{E}, \times 10)$

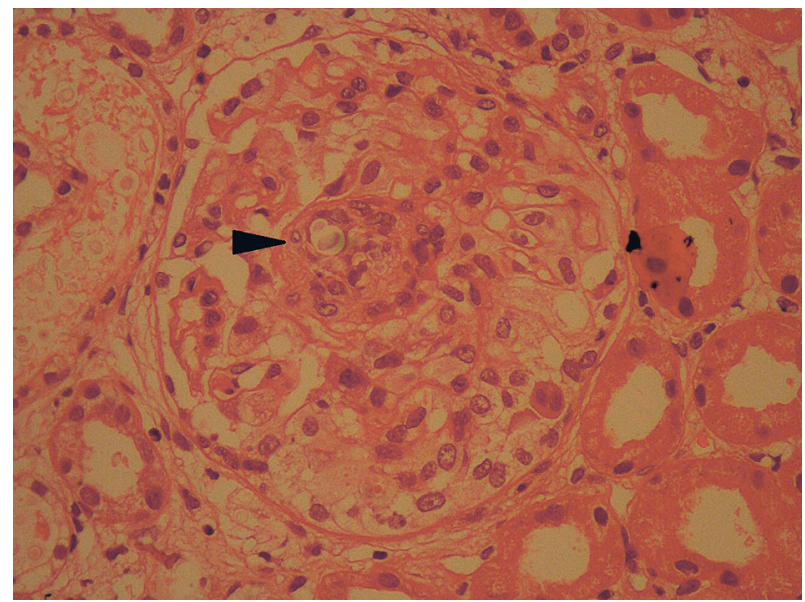

Figure 3. Same glomerulus as in Figure 1. Round yeast within the capillary can be seen (arrowhead) $(H / E, \times 40)$

enced headache, visual blurring, nausea, and vomiting and was hospitalised due to possible CMV, TBC, or HIV-related retinopathy and raised creatinine level. Cryptococcus neoformans grew in his blood culture, but CMV or TBC were negative. On day 7, yeasts consistent with Cryptococcus neoformans were isolated from the cerebrospinal fluid. His CD4 was $6 \%, \mathrm{CD} 8$ was $11 \%$, creatinine was 2.36 , and albumin was 2.2 at that time. Because of the persistent proteinuria, kidney biopsy was applied. On histological examination, some glomeruli appeared normal, while endocapillary proliferative glomerulonephritis (GN) (Figures 1, 2 and 3) was seen in other glomeruli. Tubules were enlarged with many round yeasts (Figure 4). Mucicarmine stain confirmed peripheral capsule (Figure 4, inset) [1]. Immunofluorescence staining with IgG, IgA, IgM, C3, C1q, fibrinogen, kappa, and lambda light chains revealed no deposition. Electron microscopy was not applied. The patient was well with $26 \mathrm{BUN}$ (range $8-20 \mathrm{mg} / \mathrm{dl}$ ) and 1.70 creatinine (range $0.84-1.25 \mathrm{mg} / \mathrm{dl}$ ) five months after the biopsy date.

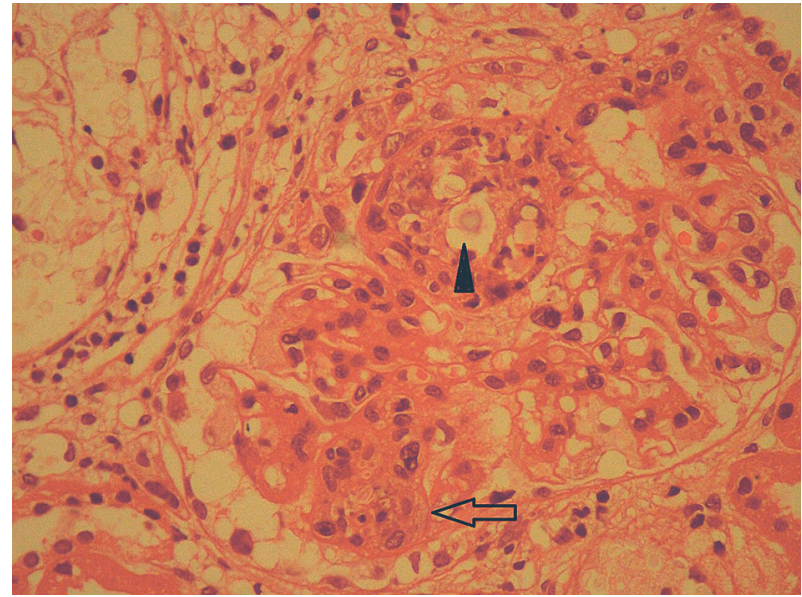

Figure 2. Same glomerulus as in Figure 1. Neutrophil leucocyte (arrow) and round yeast (arrowhead) within the capillary are easily appreciated $(\mathrm{H} / \mathrm{E}, \times 40)$

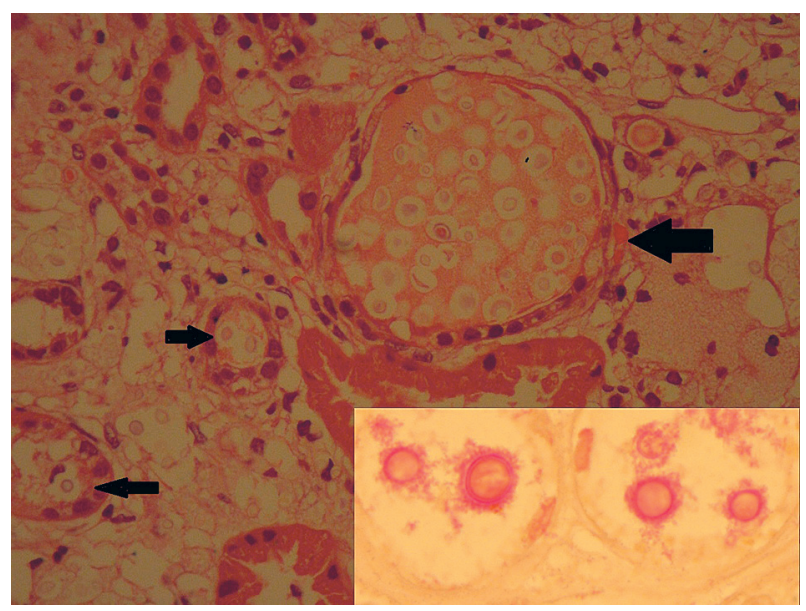

Figure 4. Tubules full of round yeast (arrows) (H/E, x40), inset: the inner capsule of the organism stains red in this photomicrograph (Mucicarmine, ×100)

\section{Discussion}

Cryptococcal infection is the fourth most common opportunistic infection in HIV+ patients. Infection with Cryptococcus is initiated by inhalation of airborne cells from the environment. From the alveoli, internalisation by endothelial cells occurs and the organisms access the bloodstream $[2,3]$.

The most common secondary immunodeficiency is caused by HIV, and although different forms of renal disease were noted in this population, HIVAN is the most common cause of renal failure in HIV1+ patients. The term HIVAN is reserved for the typical histopathological form of focal and segmental glomerulosclerosis characterised by the findings of coexistent glomerular and severe tubulointerstitial disease [4]. HIV infects cells by using CD4 molecules, and subsequent loss of CD4+ T cells is the hallmark of decreased Th1 response, which results in a profound deficiency in cellmediated immunity: the absence of CD4+ T lymphocytes 
decreases the function of antigen-presenting activity in macrophages in these patients [5].

The pathophysiology of GN includes glomerular capillary damage. In an experimental study, glomerular capillary repair was inhibited after capillary injury by administrating anti-VEGF, and decreased endothelial cell proliferation and capillary repair in glomeruli have been observed [6]. Cryptococcus and its capsular antigens induce vascular endothelial growth factor (VEGF) secretion, and VEGF levels elevate in CSF, plasma, and serum compared with healthy control subjects [7]. It has been demonstrated that CD4 (+) T lymphocytes are the cells that produce VEGF in response to cryptococcal antigens. We identify a hypothesis that decreased CD4+ T lymphocytes failed to produce VEGF in response to cryptococcal infection, and decreased VEGF diminished endothelial-cell proliferation and capillary repair in glomeruli and ended up with proliferative GN.

Proliferative GN without immunofluorescence staining and several normal glomeruli in this case support the theory that our case is not an HIV-associated immune complex GN.

One published case with cryptococcal mediastinitis and crescentic GN had relative CD4 lymphopaenia with normal CD4/CD8 ratios [8]. Another patient with rheumatoid arthritis and pulmonary cryptococcosis developed necrotising glomerulonephritis with crescents. After treatment of cryptococcosis, the crescents disappeared, which suggested that it represented pauci-immune T-cell-mediated injury related to pulmonary cryptococcosis [9].

Ibrahim et al. reported that a capsular or sparsely encapsulated organisms exhibited higher endothelial cell adherence than did encapsulated Cryptococcus [3]. Organisms isolated from the environment are small and poorly encapsulated $[10,11]$. On the other hand, organisms isolated from cerebrospinal fluid are usually heavily encapsulated [12]. It is clear that the organism probably changes the size of its capsule while disseminating. Our case's tubules were full of capsulated organism. Instead of capsular size change, disrupted capillary endothelial cells may have caused extensive leakage of the capsulated organism into the tubular area. Intraglomerular Cryptococcus was shown in the glomerulus from the HIVAN patient as well [13].

\section{Conflict of interest}

The author's declared no potential conflicts of interest with respect to the research, authorship, and/or publication of this article.

\section{References}

1. Lazcano O, Speights VO Jr, Strickler JG, et al. Combined histochemical stains in the differential diagnosis of Cryptococcus neoformans. Mod Pathol 1993; 6: 80-84.

2. Okubo Y, Tochigi N, Wakayama M, et al. How histopathology can contribute to an understanding of defense mechanisms against cryptococci. Mediators Inflamm 2013; 465319; doi: 10.1155/ $2013 / 465319$
3. Ibrahim AS, Filler SG, Alcouloumre MS, et al. Adherence to and damage of endothelial cells by Cryptococcus neoformans in vitro: role of the capsule. Infect Immun 1995; 63: 4368-4374.

4. Avila-Casado C, Fortoul TI, Chugh SS. HIV-associated nephropathy: experimental models. Contrib Nephrol 2011; 169: 270-285.

5. Shibuya K, Coulson WE, Wollman JS, et al. Histopathology of cryptococcosis and other fungal infections in patients with acquired immunodeficiency syndrome. Int J Infect Dis 2001; 5: 78-85.

6. Masuda Y, Shimizu A, Kataoka M, et al. Inhibition of capillary repair in proliferative glomerulonephritis results in persistent glomerular inflammation with glomerular sclerosis. Lab Invest 2010; 90: 1468-1481.

7. Coenjaerts FE, van der Flier M, Mwinzi PN, et al. Intrathecal production and secretion of vascular endothelial growth factor during Cryptococcal meningitis. J Infect Dis 2004; 190: 1310-1317.

8. Suárez-Rivera M, Abadeer RA, Kott MM, et al. Cryptococcosis associated with crescentic glomerulonephritis. Pediatr Nephrol 2008; 23: 827-830.

9. Nakayama M, Hori K, Ishida I, et al. A case of necrotizing glomerulonephritis presenting with nephrotic syndrome associated with pulmonary cryptococcosis. Clin Exp Nephrol 2005; 9: 74-78.

10. Farhi F, Bulmer GS, Tacker JR. Cryptococcus neoformans. IV. The not-so-encapsulated yeast. Infect Immun 1970; 1: 526-531.

11. Bulmer GS. Twenty-five years with Cryptococcus neoformans. Mycopathologia 1990; 109: 111-122.

12. Levitz SM. The ecology of Cryptococcus neoformans and the epidemiology of cryptococcosis. Rev Infect Dis 1991; 13: 1163-1169.

13. Veatch A, Dikman SH. Images in clinical medicine. Human immunodeficiency virus nephropathy and intraglomerular Cryptococcus neoformans. N Engl J Med 1998; 339: 887. 